\title{
4. Hypervolume and Topology
}

The previous chapters were incomplete in two ways. The first, which is the chief topic for the rest of this book, is that the ease or difficulty of adjoining extra structure might affect the ranking of the hypotheses. I begin that investigation in this chapter by considering topological structure. Because it is easier to characterise, this provides a foil for the problem of characterising differentiable structure, discussed in the next chapter.

I also discuss another respect in which the first three chapters were incomplete: although I was considering 4-dimensional aether the intuitions relied upon were based on those about objects extended in 3 dimensions. Yet Special and General Relativity are widely, and I think correctly, taken to imply that there is no such property as the diameter of something extended in Space and Time, for diameters are framerelative. ${ }^{77}$ Because I have relied upon intuitions about diameter I need to show that those intuitions survive transition to frame-relativity.

First, though, I reply to the objection that hypervolume and diameter are not fundamental quantities. That is, they depend ontologically on something else. I shall concentrate on hypervolume. Similar considerations apply to diameter.

\section{Hypervolume and Ockham's Razor}

One of the advantages of Point Discretion was that we replace hypervolume by another measure of quantity, the number of points, which is unproblematic. On the other hypotheses about the structure of the aether, it is tempting to posit a relation between two regions $\mathrm{u}$ and $\mathrm{v}$ and a number $\mathrm{x}$, namely that the $\mathrm{u}$ and $\mathrm{v}$ have hypervolumes in the proportion whose value is $\mathrm{x}$. This requires relations between things of different categories, namely regions and numbers, and such cross category relations should, I

Although we can define a frame-independent measure of extent it is not a diameter, because it is not an analog of the familiar, but frame-relative, 3 dimensional diameter. For it fails to satisfy the principle that if two regions overlap, then the diameter of their sum is no greater than the sum of their diameters. 
say, be avoided if we can. ${ }^{78}$ Moreover, I follow Newton in holding that numbers just are proportions, in which case the phrase 'whose value is' is redundant. ${ }^{79}$ So I say that hypervolumes are properties of regions and that these properties themselves stand in various relations of proportion, which we then identify with non-negative numbers. ${ }^{80}$ Considering volumes in three dimensions in place of hypervolume for simplicity of exposition, we may say that the volume 1 cubic metre and the volume 1 cubic foot stand in a proportion relation which is identical to some number (approximately 35.3).

Ockham's Razor is sharp when it comes to fundamental properties and relations, and I think we should posit a fairly small number of these. On my preferred account of hypervolumes a trans-categorical triadic relation is replaced by an infinity of distinct properties. So by Ockham's Razor these should not be fundamental. Yet, the objection would go, I have not explained what hypervolume depends on. Moreover, because hypervolume is not fundamental our intuitions about it are vulnerable to defeat by explication. That is, we should investigate to what extent our intuitions depend on ones concerning the more fundamental structure, and if they do, check that our intuitions survive this explication.

Except in the case of Point Discretion, hypervolume depends on the single dyadic less-than-or-equal quantity relation, $\mathrm{x} \leq \mathrm{q} \mathrm{y}$. There is a derived equal quantity relation, $\mathrm{x}=\mathrm{q} \mathrm{y}$, which holds if $\mathrm{x} \leq \mathrm{q} \mathrm{y}$ and $\mathrm{y} \leq \mathrm{q}$, and a derived less quantity relation, $\mathrm{x}<_{\mathrm{q}} \mathrm{y}$, which holds if $\mathrm{x} \leq \mathrm{q} \mathrm{y}$ but not $\mathrm{x} \leq \mathrm{q}$ y. The theory becomes more complicated if, contrary to our intuitions, there are some regions to which no hypervolume is assigned. In

One reason is that if the Xs are related to the Ys, then in addition to the categories of relations between Xs and relations between Ys, there must be an additional category of relations between Xs and Ys, which is uneconomic.

'By number we understand not so much a multitude of unities, as the abstracted ratio of any quantity to another quantity of the same kind, which we take for unity' (Newton, 1728:2). See also (Forrest and Armstrong 1987).

To be sure, we may sometimes say that the hypervolume of $u$ and the hypervolume of $\mathrm{v}$ stand in the ratio $\infty$ to 1 , but I paraphrase this as saying that the hypervolume of $\mathrm{v}$ and the hypervolume of $\mathrm{u}$ stand in the ratio 0 to 1 . 
that case having hypervolume would have to be taken as a primitive property of regions.

For regions that have hypervolume - hopefully all regions - the $\leq \mathrm{q}$ relation is transitive reflexive and linear. Linearity is here the principle that for any regions $\mathrm{x}$ and $\mathrm{y}$ that have hypervolume either $\mathrm{x} \leq \mathrm{q}$ y or $\mathrm{y} \leq \mathrm{q}$.

First let is consider the discrete case. If Point Discretion is correct, then, as already mentioned, we do not need the $\leq \mathrm{q}$ relation. For the quantity of a region is just the number of points. In the case of Extended Simples and its variants, all we need is the relation of same-quantity which we may either take to be a fundamental equivalence relation or characterise it thus: $\mathrm{x}=_{\mathrm{q}} \mathrm{y}$ if $\mathrm{x} \leq_{\mathrm{q}} \mathrm{y}$ and $\mathrm{y} \leq_{\mathrm{q}} \mathrm{x}$. For then the plausible hypothesis that all extended simples have the same hypervolume enables us to use the number of simple parts in a region as a measure of its hypervolume, showing how hypervolume depends on the $\leq \mathrm{q}$ relation. ${ }^{81}$

In the continuous case we may use the standard methods of measurement theory due to Otto Hölder to derive hypervolume from the $\leq_{\mathrm{q}}$ relation (See Suppes and Zinnes 1963). We start with the region $œ$ that we may take to have unit hypervolume. ${ }^{82}$ We may then characterise a region of positive hypervolume as a region $\mathrm{u}$ such that for some finite

If we were to posit extended simples that are not all of equal hypervolume, then we could still take hypervolume as dependent on the $\leq_{\mathrm{q}}$ relation. In that case I would assume that for any two simple regions $u$ and $v$ there are integers $m$ and $n$ and some disjoint extended simples, the Ws, all of the equal hypervolume, such that $\mathrm{u}$ is of equal hypervolume to the sum of $\mathrm{m}$ of $\mathrm{W}$ and $\mathrm{v}$ of equal hypervolume to the sum of $\mathrm{n}$ of them. There are, however perverse examples in which the purported hypervolume could not be derived from the $\leq_{\mathrm{q}}$ relation. For instance, suppose there are only finitely many regions in the whole universe, all of which are sums of extended simples, some of which have hypervolume 1 unit and some hypervolume $\sqrt{2}$ units. In such cases we could not ground the hypervolumes in the $\leq_{q}$ relation. But we have no reason to posit such perverse structures.

To avoid ending up with a system of infinite hypervolumes or infinitesimal hypervolumes we need an anthropocentric scale, taking œ to be, say, the region occupied by the standard metre during the Nineteenth Century. 
number of regions $\mathrm{x} 1$ to $\mathrm{x}_{\mathrm{n}}, \mathrm{xj}_{\mathrm{j}} \leq_{\mathrm{q}} \mathrm{u}, \mathrm{xj}_{\mathrm{j}}<_{\mathrm{q}} \propto$, and $\mathrm{x} 1+\ldots+\mathrm{x}_{\mathrm{n}}=œ$. Restricting attention to regions that have positive hypervolume, we may propose the following principles:

(1)If $x$ is a part of $y$ then $x \leq q y$.

(2)If $\mathrm{x} \leq \mathrm{q}$ y and $\mathrm{y} \leq \mathrm{q} \mathrm{z}$ then $\mathrm{x} \leq \mathrm{q} \mathrm{z}$.

(3)Either $\mathrm{x} \leq \mathrm{q}$ y or $\mathrm{y} \leq \mathrm{q} x$

(4)Given any $x$ and $y$ there is some $z$ such that $x=q z$ and $y$ and $z$ are disjoint.

(5)If $\mathrm{x} \leq \mathrm{q} y$ and $\mathrm{z}$ is disjoint from $\mathrm{x}$ and $\mathrm{y}$ then $(\mathrm{x}+\mathrm{z}) \leq \mathrm{q}(\mathrm{y}+\mathrm{z})$.

(6)If $x<_{q} y$ then there is some $z$ disjoint from $x$ such that $(x+z)={ }_{q}$ $\mathrm{y}$.

(7)If $\mathrm{x} \leq \mathrm{q} \mathrm{y}$ there is an integer $\mathrm{m}$ and pairwise disjoint regions $\mathrm{u} 1$ to $\mathrm{u}_{\mathrm{m}}$ such that $\mathrm{x}={ }_{\mathrm{q}} \mathrm{uj}_{\mathrm{j}}$ for all $\mathrm{j}$, and $\mathrm{y} \leq_{\mathrm{q}}\left(\mathrm{u}_{1}+\ldots+\mathrm{u}_{\mathrm{m}}\right)$.

From these principles it follows that there is a measure of quantity, hypervolume, taking values from 0 to $\infty$, such that (a) $\mathrm{x} \leq \mathrm{q} y$ if and only if $\operatorname{hvol}(\mathrm{x}) \leq \operatorname{hvol}(\mathrm{y})$, and (b) if $\mathrm{x}$ and $\mathrm{y}$ are disjoint, $\operatorname{hvol}(\mathrm{x}+\mathrm{y})=\operatorname{hvol}(\mathrm{x})+$ $\operatorname{hvol}(\mathrm{y}){ }^{83}$

Premise Ten may be modified to obtain Premise Ten*:

Consider some totally ordered regions the Xs. Suppose there is some region $\mathrm{w}$ such that, for any $\mathrm{x}$ that is $\mathrm{X}, \mathrm{x} \leq \mathrm{q}$ W. Then, if the Xs have a $v, v \leq \mathrm{q}$.

Premise Ten* together with (1) to (7) show that hypervolume is countably subadditive, but in any case all we required in Chapter Two was that the sum of regions of zero hypervolume is of zero hypervolume, where a region $\mathrm{x}$ is of zero volume if for all $\mathrm{y}, \mathrm{x}+\mathrm{y} \leq \mathrm{q} \mathrm{y}$, for which Premise Ten* directly suffices.

Of the eleven Premises of Chapter Two those that are required in addition to Countable Subadditivity are already of a purely qualitative

Consider the equivalence classes $u, v$ etc under ${ }_{\mathrm{q}}$. Define $\mathrm{R}$ by $u \mathrm{R} v$ iff for some $\mathrm{x} \in u, \mathrm{y} \in v, \mathrm{x} \leq_{\mathrm{q}} \mathrm{y}$. Define $u \mathrm{~S} v$ as the equivalence class of any $\mathrm{x}+\mathrm{y}$ such that $\mathrm{x} \in u, \mathrm{y} \in v$ and $\mathrm{x}$ and $\mathrm{y}$ are disjoint. Then it is easy to see that the system consisting of the equivalence classes, and the relations $\mathrm{R}$ and $\mathrm{S}$ satisfy the axioms of an extensive system (Suppes and Zinnes 1963: 42). Therefore, their representation theorem (Suppes and Zinnes 1963: 43) holds. 
nature and can be expressed easily enough in terms of the less-than-orequal quantity relation. Thus we may paraphrase 'hvol(u) $<1 / \mathrm{n}$ ' by 'There are $\mathrm{n}$ regions of equal quantity $\mathrm{x} 1$ to $\mathrm{xn}$, such that $\mathrm{u} \leq_{\mathrm{q}} \mathrm{xj}$ and such that $\mathrm{x}_{1}+\ldots+\mathrm{x}_{\mathrm{n}}=\propto . '$

\section{The threat of Relativity}

Rejecting as I do the neo-Lorentzian theories, championed by Craig (2001), I assume there is no necessarily privileged frame of reference. But diameter is frame-relative, so there is no such thing as the diameter for us to have intuitions about. Intuitions should, I submit, be stated in terms of genuine properties and relations, even if these are not fundamental, so this is a threat to some of premises used in Chapter Two.

It should be noted that a similar threat does not arise for hypervolume, which is independent of the choice of frame of reference, even in General Relativity, where it depends on the gravitational field but not the choice of coordinates.

I begin by noting that the Premises of Chapter Two concern only the following properties defined in terms of diameters, and their negations: a region having zero diameter; a region having finite but non-zero diameter and; a sequence of regions having diameters tending to zero in the limit. This last occurs whenever we consider arbitrarily small diameters. These properties, and the premises stated in terms of them, are frame-invariant. Furthermore we can explicate them using Alexandrov intervals, namely non-empty meets of future and past light cones. A region is of finite diameter if it is part of some Alexandrov interval. A region $\mathrm{u}$ is of zero diameter if: for any region $\mathrm{z}$, there is an Alexandrov interval $\mathrm{y}$ such that $\mathrm{u} \leq \mathrm{y}$ and $\mathrm{y} \leq \mathrm{q} \mathrm{z}$. Assuming $\propto$ has finite volume, the sequence of regions $\mathrm{uj}_{\mathrm{j}} \mathrm{j}=1,2, \ldots$ has diameters tending to zero in the limit if:

For every positive integer $m$, there is some positive integer $n$, and an Alexandrov interval $v_{n}$ such that $u_{j} \leq v_{n}$ if $j \geq n$, and there are $\mathrm{m}$ pairwise disjoint parts of $\propto, \mathrm{w}_{\mathrm{k}}, \mathrm{k}=1,2, \ldots, \mathrm{m}$ for which $\mathrm{v}_{\mathrm{n}} \leq \mathrm{q} \mathrm{wk}, \mathrm{k}=1$. . m.

This enables us to state the eleven Premises of Chapter Two without requiring diameters. 


\section{Mereotopology}

Even if the topological structure is not fundamental, it is part of the necessary structure of the aether and so it is of interest that it can be described simply. In Mathematics, topology only applies to a point-based theory of the aether, but for our purpose, we need to extend the idea of topology to cover point-free theories. What we need is provided by the flourishing discipline of mereotopology. ${ }^{84}$

The fundamental mereotopological concept could be taken as touching or adjacency. It sounds a little odd, however, to say that every region touches itself, or even that any two overlapping regions touch. So instead I follow the standard usage that the dyadic relation of connection holds between adjacent or overlapping regions. There is then a harmless ambiguity between this relation and topological property of being connected that holds of a region unless it is the sum of two parts that are not related by the connection relation.

Sometimes is more convenient to consider separation, the negation of connection, namely neither overlapping nor touching. Separation is definable in terms of diameters as follows. The regions $\mathrm{u}$ and $\mathrm{v}$ are separated unless there is a sequence of regions $\mathrm{x}_{\mathrm{n}}$ overlapping both $\mathrm{u}$ and $\mathrm{v}$ and the diameters of the $\mathrm{x}_{\mathrm{n}}$ tend to zero.

Interior parthood, «, may be defined in terms of separation thus:

$\mathrm{x} \ll \mathrm{y}$ if $\mathrm{x}$ is separated from every region disjoint from $\mathrm{y}$.

Assuming Boolean mereology we expect the following to hold:

1. If $\mathrm{u} \ll \mathrm{v}$ then $\mathrm{u}<\mathrm{v}$.

2. If $w \leq x \ll y \leq z$ then $w \ll z$.

3. If $u \ll x$ and $v \ll y$ then $u \vee v \ll x \vee y$.

4. If $u \ll x$ and $v \ll y$ then $u \wedge v \ll x \wedge y$.

5. If $u \ll x$ then $\neg x \ll \neg u$. 1999). 
Clearly if 5 holds we need only one of 3 or $4 .^{85}$ In addition, if we have a hypervolume measure we expect that:

6. If $\mathrm{x}$ « $\mathrm{y}$ and $\mathrm{x}$ is of finite hypervolume then $\operatorname{hvol}(\mathrm{x})<\operatorname{hvol}(\mathrm{y})$. Now we could define separation in terms of interior parthood thus: $x$ is separated from $\mathrm{y}$ if $\mathrm{x}$ is an interior part of the complement of $\mathrm{y}$. But that makes it a mystery why separation is symmetric, something that should be intuitively obvious. Hence I take separation or connection as more fundamental than interior parthood, even though the latter is more convenient theoretically. ${ }^{86}$

The mereotopology may therefore be characterised in terms of any of the easily inter-definable relations of connection, separation and interior parthood, and I hold that either connection or separation as fundamental. But there are alternative candidates for the fundamental topological item, and I need to explain why I reject them. For instance, some or all regions have the special property of being open, where an open region is one that is the sum of its interior parts. If we adjoin a fictitious empty region, $\varnothing$, that is treated as open too. In order to mimic point-set topology we would suppose the resulting lattice of open regions is a complete Heyting one, as in the theory of locales (Johnstone 1982).

The argument for not taking the property being open as fundamental is that I take it that, other things being equal, our conceptual analysis is a guide to ontological dependence. I do not think that the concept of being open is conceptually primitive. Instead it is understood in terms of some other concept such as interior parthood, whereas separation is itself highly intuitive. This argument may be strengthened by noting that we have no way of visualising or imagining in a tactile fashion the difference between open and other regions, and hence nothing for our intuitions to get a grip on. By contrast, we can imagine separation. To be sure, in a point-based theory we can imagine the difference between an

Consider the hypothesis that regions are represented by the maximal open sets of quadruples. This would be an example of a non-Boolean system in which 1, 2, 3, 4 and 6 (below) held. spect to interior parthood (Forrest 2010). 
open region and a closed one by considering two objects moving closer but incapable of overlapping. If they always occupy open regions they can touch but if they always occupy closed regions they must remain separated. But this way of getting a partial intuitive grasp of open regions only serves to support the claim that separation is a more fundamental relation.

But is topology fundamental? In the case of Minkowski Space-time and other plausible symmetric structures for the aether, such as de Sitter Space-time, we may derive the topological structure from the light-cone structure, and hence from the ordering of regions with respect to absolute priority. ${ }^{87}$ (Region $\mathrm{u}$ is absolutely prior to region $\mathrm{v}$ if with respect to every frame of reference every part of $u$ is earlier than every part of v.) On the standard, covariance interpretation of General Relativity, Spacetime is assumed to have the structure of a differentiable manifold, which, to be sure, entails that it has a topological structure. The differentiable manifold structure turns out to be problematic and it is to be hoped that it could be replaced by the topological structure, as it can be on the hypothesis of Granulated Aether (See the next chapter). It may turn out, then, that topological structure is fundamental after all.

There is another way in which topology might turn out not to be fundamental. That is if the topological structure can be replaced by mereological structure, as on the Pseudo-set Granules hypothesis discussed below, or on Sparse Continuum or any other continuous Boolean mereology in which all the regions are open. For then we construct points, and the open sets are (in addition to $\varnothing$ ) the unions of sets representing open regions.

Topology, whether fundamental or not, is important for an additional reason. It provides some additional assurance that we have not overlooked any plausible hypothesis for the necessary structure of the aether. For given a suitable topology we may construct point-locations and hence adjoin points if necessary, obtained a point-based Space-time in which the aether is located. Space-time is then a point-set topology, which is a well-researched mathematical theory. Space-time. 
There are two ways of introducing these Space-time points, here considered as fictitious aether-points. If the open regions plus $\varnothing$ form a complete Heyting lattice then we may consider maximal proper open regions as in one to one correspondence with the points, because if there were points then the set of all points except point $p$ would be a maximal proper open region. The further principle that if $u$ and $v$ overlap the same open regions then $\mathrm{u}=\mathrm{v}$, then shows that every region is the sum of real or fictitious, points. If we started with a point-based aether with a topological structure, then this procedure gives us the topology we started off with. (See Johnstone 1982.)

The second method relies upon a mereotopology characterised using connection, separation or interior parthood. We characterise the points of Space-time so as to represent regions by sets of points. Then connected regions are to be represented by sets whose closures overlap ${ }^{88}$. The method, pioneered by Peter Roeper (1997), is to take the points to be ultrafilters with respect to interior parthood. This is an explication of Whitehead's Russian doll construction of points as a sequence of ever smaller regions. $^{89}$

A noteworthy feature of the ultrafilter construction is that in the case of point-based aether with points corresponding to all quadruples of real numbers the ultrafilter construction would adjoin a fictitious point at infinity. Likewise if we started with a gunk theory in which regions were represented by suitable sets of quadruples the ultrafilter construction would adjoin a point at infinity. In both cases the point at infinity corresponds to the ultrafilter that has as its members all the complements of regions of finite diameter.

This is a modification of Marshall Stone's method of representing Boolean algebras topologically (Stone, 1936). For more details on constructing points as a ultrafilters with respect to interior parthood see (Forrest 2010). With care we may avoid assuming the Axiom of Choice.

A filter is a set of regions $\mathrm{X}$ such that: (1) If $\mathrm{x} \in \mathrm{X}$ and $\mathrm{x}$ « $\mathrm{y}$, then $\mathrm{y} \in \mathrm{X}$; and (2) If $\mathrm{x} \in \mathrm{X}$ and $\mathrm{y} \in \mathrm{X}$ then for some $\mathrm{w}, \mathrm{w} \in \mathrm{X}, \mathrm{w}$ « $\mathrm{X}$ and $\mathrm{w}$ « $\mathrm{y}$. (Note: we do not treat $\varnothing$ as a region.) A proper filter is one that does not have every region as a member. An ultrafilter is a filter that is maximal among proper filters. 
Set-theoretic constructions are not the right sort of thing to be considered points. So if aether is point free and Space-time is dependent on the aether and made up of points, then I propose that the points of Spacetime are suitable properties of regions. I anticipate the retort that properties are no more suited to be points than are sets. Strictly speaking that is correct, and I should replace points by point locations. To a fictitious point $\mathrm{p}$ there corresponds an ultrafilter of regions $\mathrm{X}$. Therefore the property of being at $p$ may be identified with the conjunction of the relational properties: overlapping $u$, for all regions $\mathrm{u}$ in $\mathrm{X}$. The property being located at the point at infinity is then just the property of being of infinite extent.

\section{Topology in the discrete case}

If Point Discretion holds, then connection collapses to overlapping, separation collapses to being disjoint, interior parthood collapses to parthood, and all regions are open. So the topology is trivial. Moreover it is compatible with any metric. This is, however, something more to be said. For there might well be a minimum non-zero value that diameters take, and in the simplest case all diameters will be a whole number times that minimum. Then we may describe points that are the minimum nonzero distance apart as neighbours, and call the sum of a point $\mathrm{p}$ and its neighbours the neighbourhood of $\mathrm{p}$. Many concepts such as an analog of the property of connectedness can be defined in terms of neighbourhoods. That would be great if the regions had frame-invariant diameters. But they do not. Instead we may posit a light cone structure analogous to that for Minkowski Space-time. In that case there is a relation of absolute priority between any points $\mathrm{u}$ and $\mathrm{v},(\mathrm{u} \prec \mathrm{v})$, where point $\mathrm{u}$ is absolutely prior to point $\mathrm{v}$ just in case $\mathrm{u} \neq \mathrm{v}$ and $\mathrm{u}$ is part of every past light cone containing $\mathrm{v}$. Then $\mathrm{x}$ is the immediate predecessor of $\mathrm{z}(\mathrm{x} \prec * \mathrm{z})$, if $\mathrm{x} \prec \mathrm{z}$, but there is no $\mathrm{y}$ such that $\mathrm{x} \prec \mathrm{y} \prec \mathrm{z}$. We may then say that points $\mathrm{x}$ and $\mathrm{z}$ are neighbours if $\mathrm{x} \neq \mathrm{z}$ and either $\mathrm{x} \prec^{*} \mathrm{z}$ or $\mathrm{z} \prec^{*} \mathrm{x}$, or if for some points $\mathrm{u}$ and $\mathrm{v}$, such that $\mathrm{u} \prec^{*} \mathrm{x} \prec^{*} \mathrm{v}$ and $\mathrm{u} \prec^{*} \mathrm{z} \prec^{*} \mathrm{v}$. We should, however, be suspicious of this topology-analog. For suppose we represent points as quadruples of integers. Then the points represented by $<0,0,0$, $0>$ and by $<\mathrm{m}, \mathrm{n}, \mathrm{p}, \mathrm{q}>$ can be immediate neighbours even though $\mathrm{m}, \mathrm{n}$, 
$\mathrm{p}$ and $\mathrm{q}$ are all large positive numbers. This peculiar feature forms the basis of the objection to Point Discretion presented in Chapter Seven.

If the aether is granulated, the characterisation of topology in terms of diameter fails. The reason is that Arbitrary Fine Division does not hold and hence the distance between extended simple regions may not be characterised as the greatest lower bound of the diameters of overlapping regions. Fortunately in these theories the idea of two regions touching is still highly intuitive. Formally it is like the topology-analog for Point Discretion with adjacency replacing being neighbours. Moreover, the topology of extended simples is frame-invariant. For change of frame will change the shape of the sets representing regions, but not the adjacency. Likewise, the shape of the granule itself is frame-invariant, being characterised in terms of adjacency.

The Pseudo-set Granules hypothesis, although more complicated than Extended Simples, does have one great advantage, namely that we can characterise the topology in mereological terms, with connection defined as overlapping. The reason is that two granules that would be nonoverlapping but adjacent on the Extended Simples hypothesis correspond, on the Pseudo-set Granules hypothesis, to atoms that have a common part, namely a shared facet, face, edge or vertex. Because the mereology is non-standard we obtain a non-trivial topology by identifying overlap with connection. In particular, the sum of two overlapping granules is a connected region (because of the failure of Weak Supplementation).

\section{The characterisation problem}

In the previous chapters I presented a variety of hypotheses about the aether by means of their coordinate representations. I require, however, a frame-independent characterisation, and one that does not involve settheoretic constructions. That is straightforward in the case of Point Discretion - ignoring fine structure we may characterise that hypothesis by saying that a region of finite diameter is the sum of finitely many parts, each of which has zero (or infinitesimal) diameter.

The case of granulated aether is almost as straightforward. A granule may be defined as a region that cannot be decomposed as the sum of two regions of lesser but positive quantity. So we begin by hypothesis- 
ing that any region of finite diameter is part of a finite sum of granules. In the case of Extended Simples every granule is simple and any region of finite diameter is the sum of disjoint granules. All that remains is to note their shape based on the primitive relation of adjacency. Variants such as Pseudo-set Granules are more complicated to describe, but the description provided in Chapter One meets the requirements of a characterisation independent of set-theoretic construction or coordinate representation.

In the case of a continuous point-based hypothesis it suffices to characterise the topological space these points form, together with some way of distinguishing regions of positive measure from those of zero measure. For then the description of the hypotheses in these terms proceeds as in the previous chapters.

Although topological spaces occur in bewildering variety those that current physics might take to be continuous Space-time are manifolds of 4 (or some other specified finite number N) dimensions. ${ }^{90}$ Manifolds are topologically connected spaces that are locally represented by coordinate quadruples (or more generally N-tuples) of real numbers in the right way. We may think of the aether as the sum of overlapping globules, the $\mathrm{J}$, each represented by coordinate quadruples. How the globules thus represented fit together is considered in the sub-discipline of algebraic topology, where the, now proven Poincare' Conjecture, solves the characterisation problem, provided we can characterise the (mereo)topology of the globules. ${ }^{91}$ Here I ignore the hypervolume, not for any principled reason, but merely because it neither helps nor hinders the solution of the characterisation problem.

The most straightforward case, in which the manifold lacks a boundary, is that in which for any $\mathrm{j}$ in $\mathrm{J}$ the topological space of point parts of $\mathrm{j}$ is homeomorphic (i.e. topologically equivalent) to the set of

If the whole physical universe is the sum of disconnected universes the aether could, of course, be a topological space that is the sum of disjoint manifolds. I grant that possibility, but for sake of exposition I concentrate on one universe.

Grigori Perelman proved the case of three dimensions. The cases of more than three, and in particular four, dimensions had already been proven. 
quadruples of real numbers endowed with the usual topology. That is an extrinsic characterisation, setting up the problem of finding an intrinsic one, or at least one that does not resort to coordinates. The only way to do this that I know of is to rely on the existence of symmetries (automorphisms), in this case homeomorphisms from the topological space onto itself. ${ }^{92}$ Let $\mathrm{T}$ be the topological space whose points are the point parts of $\mathrm{j}$. Then I require that there be a set $\mathrm{F}$ of homeomorphisms of $\mathrm{T}$ onto $\mathrm{T}$ such that $(0)$ to $(5)$ hold:

0 . If $f$ and $g$ are in $F$ and if, for every point part $x$ of $j, f(x)=g(x)$, then $\mathrm{f}=\mathrm{g}$.

Because homeomorphisms are mappings and mappings are purely formal relations, the condition (0) is strictly redundant.

1. If $\mathrm{f}$ is in $\mathrm{F}$, its inverse $\mathrm{f}-1$ is also in $\mathrm{F}$.

2. If $f$ and $g$ are in $F$, then the composite $f \circ g$ is in $F$.

If (1) and (2) hold, then F is a group, and we may derive

3. The identity automorphism, Id, which maps each point part of $j$ to itself, is in F.

$\mathrm{F}$ is a commutative group if:

4. For any $f$ and $g$ in $F, f \circ g=g \circ f$.

Such a group of automorphisms $\mathrm{F}$ is said to be transitive if:

5. For any point parts $\mathrm{u}$ and $\mathrm{v}$ of $\mathrm{j}$, there is an $\mathrm{f}$ in $\mathrm{F}$, such that $\mathrm{f}(\mathrm{u})=\mathrm{v}$.

It follows from $(0)$ to $(5)$ that, for any point part of $j, u, f(u)=u$ only if $\mathrm{f}=\mathrm{Id}$.

If there is a set of automorphisms, F, satisfying (0) to (5), then for any point part $u$ of $j$ there is a one to one correspondence between the members of $F$ and the point parts of $j$, where $f$ corresponds to $f(u)$. We may therefore characterise the topological space T made up of the pointparts of $\mathrm{j}$ as homeomorphic to the quadruples of real numbers by providing conditions on commutative group $\mathrm{F}$ that suffice to show the group is 
isomorphic to the group of quadruples of reals with addition as composition. $^{93}$

Is this a satisfactory characterisation? In the Introduction I noted that reliance on a group of symmetries to characterise the structure of the aether might be unacceptable to some nominalists. There is a further difficulty in this case, however, because there are many such groups. Unless the aether has additional structure we cannot talk of the group of symmetries. Intuitively that detracts from the suitability of this way of characterising a manifold. If there were not much more serious problems characterising differentiable structure, discussed in the next chapter, this would be worth arguing about.

The characterisation of the structure of point-free aether is similar, but, I regret to say, even more complicated. The automorphisms are now mappings from the set of the parts of $\mathrm{j}$ onto itself that preserve both the mereology and the topology. The word 'part' replaces the phrase 'point part' in (0), and in the derived result (3). We might try replacing (5) by the following transitivity analog:

$5^{*}$. For any parts $u$ and $v$ of $j$, there is some member of $F, f$, such that $\mathrm{f}(\mathrm{u})$ overlaps $\mathrm{v}$.

In the point-based case $\left(5^{*}\right)$ implies (5) because points are regions. But if there are no points then $\left(5^{*}\right)$ is a weaker condition. For suppose we construct point locations in such a way that for each region $u$ there is a set $U$ of such point locations. (We exclude the 'point at infinity'.) Then $\mathrm{F}$ acts as a group of homeomorphisms from $\mathrm{U}$ onto itself. The conditions

By requiring the one to one correspondence between $\mathrm{T}$ and the group $\mathrm{F}$ to be a homeomorphism we may consider $\mathrm{F}$ to be a topological group. That is, it is itself a topological space and the operations of composition and taking inverses are continuous. Because $\mathrm{F}$ is a topological group the characterisation problem is then easily solved. The following conditions, for instance, are jointly sufficient for $\mathrm{F}$ to be isomorphic as a topological group to the $\mathrm{N}$ tuples of real numbers with addition as the group operation.

1. $\mathrm{F}$ is a commutative topological group.

2. F is a locally compact, non-compact, connected space of topological dimension $\mathrm{N}$, with a countable dense set.

3. Apart from the trivial subgroup $\{\mathrm{Id}\}, \mathrm{F}$ has no subgroup with compact closure. 
(0) to (4) and ( $\left.5^{*}\right)$ are not enough to ensure transitivity. For given a point location $\mathrm{x}, \mathrm{F}(\mathrm{x})=\{\mathrm{f}(\mathrm{x})$ : $\mathrm{f}$ is an $\mathrm{F}\}$ is not necessarily the whole of $\mathrm{U}$. Instead all we may assume is that $\mathrm{F}(\mathrm{x})$ is dense, that is $\operatorname{cl}(\mathrm{F}(\mathrm{x}))=\operatorname{cl}(\mathrm{U})$.

Because of the replacement of (5) by $\left(5^{*}\right)$, I shall require the aether to be the sum of globules, the Ks, that are interior parts of other globules, the Js. Consider $\mathrm{k}$ « $\mathrm{j}$. Then the point locations for $\mathrm{j} \in \mathrm{J}$ form a set $\mathrm{V}$, those for $\mathrm{k} \in \mathrm{K}$ a set $\mathrm{U}$ and $\mathrm{cl}(\mathrm{U}) \subseteq \mathrm{V}$. In place of $\left(5^{*}\right)$ I require:

$5^{* *}$. For every $\mathrm{k}$ in $\mathrm{K}$ there is some compact set $\mathrm{W}$ of Fs, such that:

(a) for any parts $u$ and $v$ of $k$, there is some $f$ in $W$, such that $\mathrm{f}(\mathrm{u})$ overlaps $\mathrm{v}$, and (b) there is no proper subgroup of $\mathrm{F}$ that contains W. ${ }^{94}$

This shows that the associated action of $\mathrm{F}$ on the point locations is transitive, as required.

\section{Conclusions about hypervolume and topology}

1. Except in the case of Point Discretion where we do not need it, hypervolume may be taken to depend on a linear ordering, namely being of no-less-quantity-than.

2. Special and General Relativity cast doubt on the occurrence of frameindependent diameters. This does not threaten our intuitions about diameter because we may explicate the intuitions using the frameindependent Alexandrov intervals.

3. If topological structure is required then I note that Pseudo-set Granules have a non-standard mereology upon from which the topology could be derived. Absent some other structure, such as absolute priority on which topology could be grounded that is an advantage for those that hypothesis.

4. If the aether has no structure in addition to the measure of quantity and the topology, then the characterisation problem is solved in the

Because of the conditions stated in the previous footnote, (b) is redundant. Also note that (a) could not hold unless the set of all point locations for $\mathrm{k}$ in $\mathrm{K}$ has compact closure. By the Heine-Borel theorem the fact that we will eventually have a representation using coordinate N-tuples shows that this will be the case provided no $\mathrm{k}$ in $\mathrm{K}$ has the property of being of infinite extent. 
case of granulated aether hypotheses. But in the continuous case, it lacks any entirely satisfactory solution. Ideally we would characterise the aether by noting characteristics of the whole group of symmetries (automorphisms) rather than considering some transitive commutative subgroup. But that requires more structure. In the point-free case the characterisation is rather more complicated. I shall later be arguing that a tenable hypothesis of continuous aether requires the 'reactionary' supposition that the aether is highly symmetric. The characterisation problem for the continuous aether will not therefore have to be solved in the context of topology and quantity alone. Hence, the not entirely satisfactory solutions of the last section will no longer be required. 